\title{
MicroRNA-183/182/96 cooperatively regulates the proliferation of colon cancer cells
}

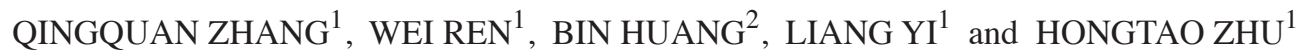 \\ ${ }^{1}$ Department of General Surgery, The 15th Hospital of People's Liberation Army, Wusu, Xinjiang 833000; \\ ${ }^{2}$ Department of General Surgery, Aoyoung Hospital, Zhangjagang, Jiangsu 215600, P.R. China
}

Received January 1, 2014; Accepted October 24, 2014

DOI: $10.3892 / \mathrm{mmr} .2015 .3376$

\begin{abstract}
The microRNA (miR/miRNA)-182/183/96 cluster comprises miR-96,-182 and -183 . The present study examined five previous microarray-based human colon cancer miR expression profiling studies and the expression of these three miRs was found to be upregulated in colon cancer tissues. Subsequently, in vitro assays were performed to determine the role of the miR-183/182/96 cluster in colon cancer cells. The results demonstrated that inhibiting miR-183, miR-182 or miR-96 with antisense oligonucleotide (ASO)-mimics inhibited the proliferation of colon cancer cells. Notably, further investigation revealed that inhibiting their expression simultaneously led to a more efficient reduction in cancer cell proliferation. These results suggested that miR-182/183/96, which resides in clusters in the genome, functioned synergistically in colon cancer and implied that co-expression of the miR cluster ASOs was efficient in reducing tumorigenesis, offering novel insight into the use of miRNAs in tumor therapy.
\end{abstract}

\section{Introduction}

The survival rate of patients with colorectal cancer (CRC), which is one of the most common types of malignancy and the third leading cause of cancer-associated mortality worldwide, is delineated by a high rate of recurrence (1-3). Mutations in certain tumor-suppressor genes and oncogenes have been identified, including adenomatous polyposis coli, deleted in colorectal cancer, mothers against decapentaplegic homolog 2 (Smad2), tumor protein 53 and kirsten rat sarcoma viral oncogene homolog (4-6). These mutant genes have been used in CRC therapy; however, their treatment effectivity is limited. Therefore, further investigation of novel targeted therapeutics for the treatment of CRC is required.

Correspondence to: Mr. Qingquan Zhang, Department of General Surgery, The 15th Hospital of People's Liberation Army, 1 Changzhen Road, Wusu, Xinjiang 833000, P.R. China

E-mail: zhangqingquan1100@163.com

Key words: microRNA, microRNA-182, microRNA-96, microRNA-183, colon cancer
Previous studies have revealed that micro (mi)RNAs can regulate tumor development by targeting their downstream genes and have been identified as a novel mechanism which contributes to the pathogenesis of CRC (7-9) has been identified. These small RNAs, which are aberrantly expressed in various types of cancer, act as oncogenes or tumor suppressors and are thus candidate targets for cancer therapy.

In the present study, the results of five microarray-based human colon cancer microRNA expression profiling studies were examined (10-15), comparing colon cancer tissue with normal tissue. The results demonstrated that the miR-183/96/182 cluster was upregulated in the colon cancer tissues. The miR-183/96/182 cluster contains three members: miR-183, miR-96 and miR-182. It has been reported that these miRNAs are located within a distance of $4 \mathrm{~kb}$ from each other on the mouse chromosome 6qA3 and are transcribed in the same direction. They are expressed coordinately and are important in the sensorineural fates of cells in the mouse inner ear $(10,11)$. This miRNA cluster also has a significant role in the maintenance and survival of hair cells and post-mitotic photoreceptors of the retina (12-14). In order to examine the roles of these miRNAs in the pathogenesis of CRC in the present study, a microarray-based miRNA expression profiling study was performed to compare miRNA expression levels in colon cancer and normal tissues. Furthermore, the present study aimed to investigate the importance of miR-183/182/96 in the proliferation of CRC cells using ASO-based miRNA inhibitors.

\section{Materials and methods}

Cell culture. HT-29 and LoVo human colon cancer cell lines were purchased from the American Type Culture Collection (Manassas, VA, USA). The cells were maintained in Dulbecco's modified Eagle's medium (DMEM; Life Technologies, Carlsbad, CA, USA) with $10 \%$ fetal calf serum (FCS; Sigma-Aldrich), $100 \mathrm{U} / \mathrm{ml}$ penicillin and $100 \mu \mathrm{g} / \mathrm{ml}$ streptomycin (Life Technologies). All the cells were maintained at $37^{\circ} \mathrm{C}$ under an atmosphere of $5 \% \mathrm{CO}_{2}$ and $95 \%$ air.

Patient samples. A total of eight paired human colon cancer tissue and corresponding adjacent normal tissue samples were obtained from randomly selected cancer patients at the Department of General Surgery, The 15th Hospital of People's Liberation Army (Xinjiang, China) and all of the diagnoses 
were pathologically confirmed. Written informed consent was obtained from each patient involved in the present study prior to surgery and all procedures were reviewed by the Joint Ethics Committee of the 15th Hospital of People's Liberation Army and performed in accordance with national guidelines.

Literature search for studies that examined the expression of miR-183/96/182 in colon cancer tissues. The Pubmed database (http://www.ncbi.nlm.nih.gov/pubmed) was searched to identify eligible studies that determine the association between miR-183/96/182 and colon cancer. Search terms, including 'miR-183', 'miR-96', 'miR-182' and 'colon cancer' were used for the literature search. The selected studies met the requirement that the expression levels of miR-183/96/182 were quantitated in human colon cancer and normal tissues. The clinical characteristics of these studies were extracted and the fold changes of the expression levels of the three miRNAs in colon cancer tissues were then compared with those in normal tissue.

Reverse transcription-quantitative polymerase chain reaction $(R T-q P C R)$ analysis. Total RNAs were extracted from the tissues using TRIzol reagent (Invitrogen Life Technologies, Carlsbad, CA, USA), and miRNA were reverse transcribed using the miRCURY LNATM Universal cDNA Synthesis kit II (Exiqon, Vedbak, Denmark). RT-qPCR was then performed on an ABI Prism 7900 Sequence Detection system (Applied Biosystems, Foster City, CA) in a $10 \mu \mathrm{l}$ PCR reaction mix, including $0.67 \mu \mathrm{l}$ RT product, 1X SYBR Green PCR master mix (Invitrogen) and $1 \mu \mathrm{l}(25 \mathrm{ng})$ of both forward and reverse primers. The reactions were incubated in triplicates in a 96-well optical plate at $95^{\circ} \mathrm{C}$ for $10 \mathrm{~min}$, followed by 40 cycles of $95^{\circ} \mathrm{C}$ for $15 \mathrm{sec}$ and $60^{\circ} \mathrm{C}$ for $1 \mathrm{~min}$. U6 snRNA levels were used as an endogenous control. The primers used for the RT-qPCR were synthesized by Invitrogen Life Technologies as follows: Forward: 5'-GCGCCTATGGCACTGGTAGAA-3' and reverse: 5'-TGCAGGGTCCGAGGTATTCG-3' for miR-183; forward: 5'-TTTGGCACTAGCACAT-3' and reverse: 5'-GAGCAGGCTGGAGAA-3' for miR-96; forward: 5'-CGGCGGTTTGGCAATGGTAGAACT-3' and reverse: 5'-CCAGTGCAGGGTCCGAGGTAT-3' for miR-182; and forward: 5'-CGCTTCGGCAGCACATATACTA-3' and reverse: 5'-CGCTTCACGAATTTGCGTGTCA-3' for U6 snRNA. Data processing was conducted using the SDS software (v2.1) (Applied Biosystems) and the expression levels of the miRNAs were then calculated using the $2^{-\Delta \Delta c t}$ method after normalized to the levels of U6snRNA.

MTT assay. The synthesized RNA duplexes of antiscrambled (ASO-miR-NC), ASO-miR-183, ASO-miR-182 and ASO-miR-96 mimics were obtained from GeneChem (Shanghai, China). Following transient transfection of miRNA inhibitors, the HT-29 or LoVo cells were seeded into 96-well plates at 1,500 cells/well and MTT (Sigma-Aldrich, St. Louis, MO, USA) assays were performed daily for $72 \mathrm{~h}$. In this assay, the medium was replaced with fresh medium containing $0.5 \mathrm{mg} / \mathrm{ml}$ MTT for $4 \mathrm{~h}$ and then carefully removed. Subsequently, $150 \mu \mathrm{l}$ dimethyl sulfoxide (Sigma-Aldrich) was added to each well and mixed for $10 \mathrm{~min}$, and the optical density at $490 \mathrm{~nm}$ was determined using an enzyme linked immunosorbent assay reader (BioTek Instruments, Winooski, VT, USA). With the MTT we designed eight groups (A-H), which contained ASO-miR-183, ASO-miR-96, ASO-miR-182, either alone or in combinations of two or all three, as well as a ASO-miR-NC control group.

Colony formation assay. The cells were seeded into a 12-well plate at a density of 200 cells/well following transfection. The medium was changed every 3 days. After $\sim 10$ days, the majority of the cell clones contained $>50$ cells. The colonies were then washed with $1 \mathrm{X}$ phosphate-buffered saline and stained with crystal violet (Fisher Scientific, Pittsburgh, PA, USA) for $\sim 5 \mathrm{~min}$. Finally, images of the colonies were captured using a Nikon Eclipse E800 microscope (Nikon, Tokyo, Japan) and the number of colonies was counted. The colony formation rate $(\%)=$ (number of clones) $/$ (number of seeded cells) x 100 .

Western blot analysis. Western blot analysis was performed, as previously described (15). Rabbit polyclonal anti-Ki-67 (ab15580) and Rabbit polyclonal anti p- protein kinase B (Akt; ab66138) were obtained from Abcam (Cambridge, MA, USA). Mouse monoclonal antibodies against $\mathrm{Bcl} 2$-associated $\mathrm{X}$ protein (Bax; sc-20067) and p53 (sc-126) and horseradish peroxidase conjugated goat anti-mouse IgG (sc-2005) and goat anti-rabbit IgG (sc-2004) were purchased from Santa Cruz Biotechnology, Inc. (Dallas, TX, USA). The membranes were incubated with primary antibodies at $4{ }^{\circ} \mathrm{C}$ overnight, followed by incubation with horseradish peroxidase-conjugated secondary antibodies. Proteins were then detected using an enhanced chemiluminescence kit (GE Healthcare Life Sciences, Piscataway, NJ, USA). LabWork 4.0 software was used to measure the band intensities of the blots.

Statistical analysis. All data are expressed as the mean \pm standard deviation. The difference between groups was determined using two-tailed Student's t-test. Statistical analyses were performed using Micrsoft Excel 2013 (Microsoft Corp., Redmond, WA, USA). $\mathrm{P}<0.05$ was considered to indicate a statistically significant difference.

\section{Results}

All miR-183/96/182 cluster members are upregulated in colon cancer. The miR-183/96/182 cluster was located on the region of human chromosome $7 \mathrm{q}$ and the miRNAs were transcribed in the same direction. It has been suggested that this cluster is unregulated in colon cancer tissues. To confirm the expression levels of the members of this gene cluster in colon cancer, a total of five previous studies (Table I) (16-20), which investigated miRNA expression in colon cancer, were examined and the fold changes of these three miRNAs in colon cancer tissues were compared with those in normal tissue. The clinical characteristics of these studies were extracted and are listed in Table I. All three miRNAs had 2 -fold changes in the colon cancer tissues according to the five microarray results. To confirm these findings, RNAs were extracted from eight colon cancer samples with paired adjacent normal colon tissues and these were analyzed by RT-qPCR (Fig. 1A-C). Consistent with the Table I data, the results demonstrated that miR-183, miR-96 and miR-182 were expressed at relatively high levels in the colon cancer tissues. 


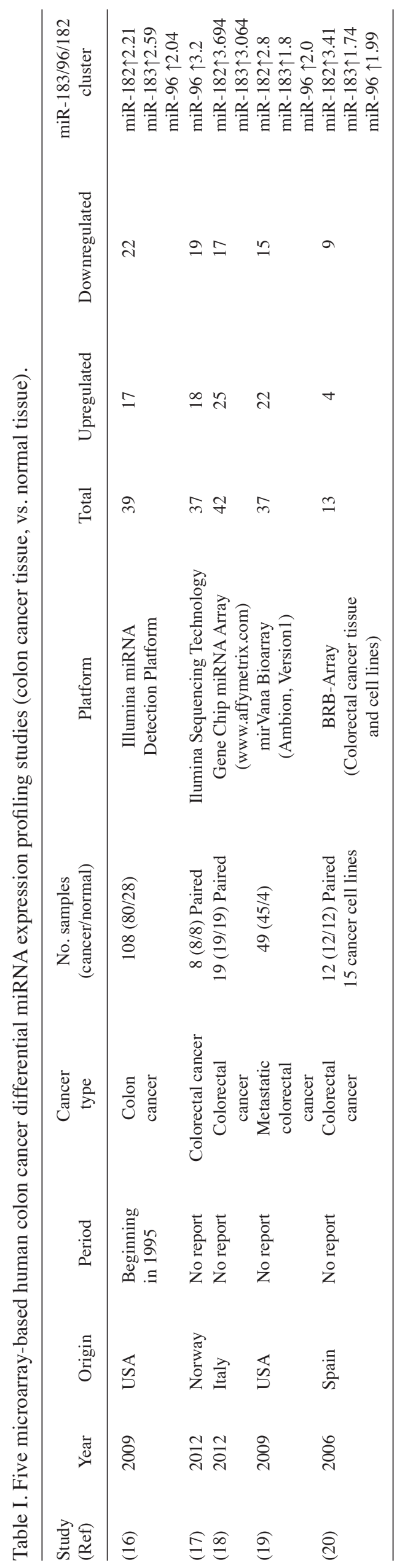

Simultaneous knockdown of the expression of the miR-183/96/182 cluster efficiently inhibits HT-29 and LoVo cell viability compared with inhibiting the miRs alone. It has been demonstrated that miR-183, 96 and 182 act as tumor oncogenes based on their high expression levels in colon cancer tissues (21). Therefore, the present study used ASO-miRs to knockdown the expression of the miR-183/96/182 cluster. It has also been suggested that miR-183, miR-96 and miR-182 have similar sequences and are highly conserved across species, therefore, raising the question of whether the three miRNAs acted coordinately or competitively to regulate the growth phenotype of colon cancer. To address this question, the present study designed eight groups, defined as groups A-H, of ASO-miRNAs containing different concentrations of ASO-miR-183, ASO-miR-96, ASO-miR-182 and ASO-miR-NC, either alone or in combination (Fig. 2). Subsequently, these ASO mimics were transfected into the HT-29 cells and the cell viability was measured using an MTT assay. As Fig. 2B shows, the HT-29 cell viability was classified into four levels. Arbitrary knockdown of two members of the miR-183/96/182 cluster (level 3) caused a reduction in HT-29 cell viability compared with the arbitrary knockdown of each alone (level 2). Furthermore, knockdown of all three members of the miR-183/96/182 cluster (group H) efficiently inhibited HT-29 cell viability (level 4) compared with the others (groups A-G). The same results were observed in the LoVo cells (Fig. 2C). These results suggested that simultaneous knockdown of the expression of the miR-183/96/182 cluster efficiently inhibited colon cancer cell viability.

Simultaneous knockdown of the miR-183/96/182 cluster expression efficiently inhibits HT-29 cell colony formation ability compared with inhibition of the miRs alone. In the present study, an MTT assay was used to detect the colon cancer cell viability $72 \mathrm{~h}$ (Fig. 2B and C) after transfection with the ASO-miRNAs. To further confirm that simultaneous knockdown of all the members of the miR-183/96/182 cluster efficiently inhibited colon cancer cell viability compared with knockdown of the miRs alone, a colony formation assay was performed. According to the design of the MTT assay, eight transfection groups were used. As shown in Fig. 3A, the colony formation rates of the HT-29 cells transfected with ASO-miR-183, ASO-miR-96 or ASO-miR-182 (groups A, B, C and D) were lower compared with ASO-miR-NC (A). In addition, the cells simultaneously transfected with ASO-miR-183 and ASO-miR-96, ASO-miR-183 and ASO-miR-182 or ASO-miR-182 and ASO-miR-96 (groups E, F and G) had a higher colony formation rate compared with those simultaneously transfected with miR-183, miR-96 and miR-182 (group H). These results were consistent with those of the MTT assay, which demonstrated that simultaneous knockdown of the expression of the miR-183/96/182 cluster efficiently reduced HT-29 cell proliferation compared with inhibition of the miRs alone.

Simultaneous knockdown of the expression of the miR-183/96/182 cluster efficiently regulates key proliferation of colon cancer cells and expression of the apoptotic protein marker. To investigate the effect of simultaneous knockdown of the expression of the miR-183/96/182 cluster on the proliferation/apoptotic signaling pathway, Ki-67, phosphorylated 

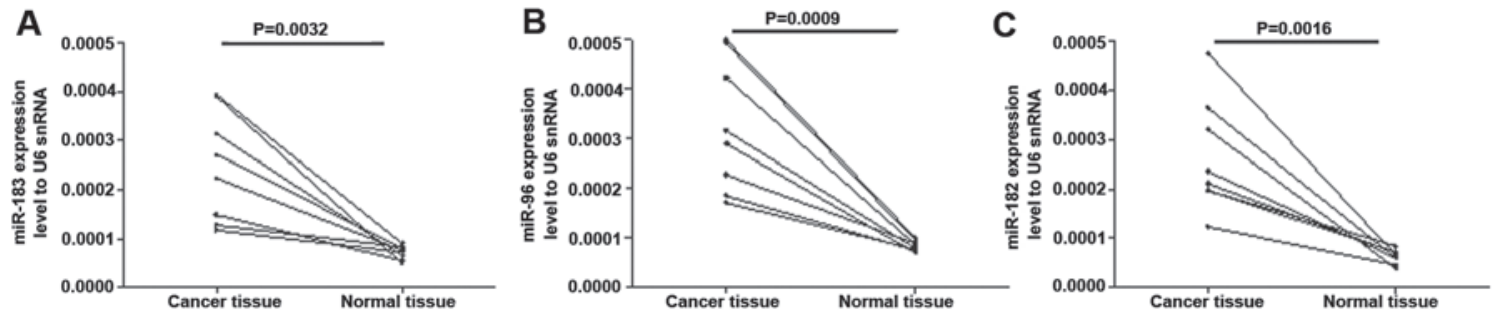

Figure 1. miR-183/96/182 were upregulated in colon cancer tissues. Expression levels of (A) miR-183, (B) miR-96 and (C) miR-182 were analyzed by reverse transcription quantitative polymerase chain reaction in eight paired colon cancer tissues and adjacent non-tumor tissues. U6snRNA was used as a control $\mathrm{P}<0.05$ was considered to indicate a statistically significant difference.

A Eight groups of transfection oligonucleartide.
\begin{tabular}{|c|c|c|c|c|c|c|c|c|}
\hline & A & B & C & D & E & F & G & H \\
\hline ASO miRNA NC & 1.2 & - & - & - & - & - & - & - \\
\hline ASO miR-183 mimics & - & 1.2 & - & - & 0.6 & 0.6 & - & 0.4 \\
\hline ASO miR-182 mimics & - & - & 1.2 & - & 0.6 & - & 0.6 & 0.4 \\
\hline ASO miR-96 mimics & - & - & - & 1.2 & - & 0.6 & 0.6 & 0.4 \\
\hline
\end{tabular}

$(\mu \mathrm{mol})$
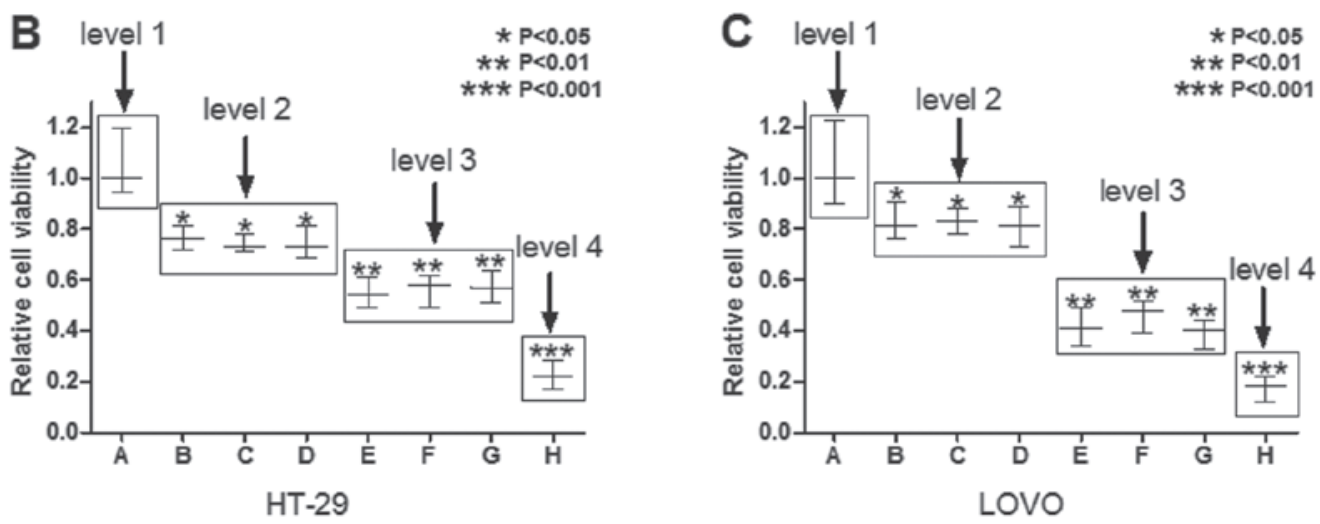

Figure 2. Effects of simultaneous knockdown of miR-183/96/182 on the cell viability of the HT-29 and LOVO cells. (A) Eight groups comprising different concentrations and types of ASO-miR mimics were designed for colon cancer cell transfection, defined as groups A-H. The dose of these mimics were measured in $\mu \mathrm{mol}$. (B) An MTT assay was performed to detect the effects of the different ASO-miRNA transfection groups on the cell viability of the HT-29 cells $72 \mathrm{~h}$ after transfection. The cell viability in group A was normalized to 1. (C) A similar MTT assay was performed for the LoVo cells. All the assays were repeated three times and all the data are expressed as the mean \pm standard deviation. Differences between groups were determined by two-tailed Student's t-test. ${ }^{*} \mathrm{P}<0.05,{ }^{* *} \mathrm{P}<0.01$ and ${ }^{* * *} \mathrm{P}<0.001$ vs. group A. miR, microRNA; ASO, antisense oligonucleotide; NC, negative control.

A
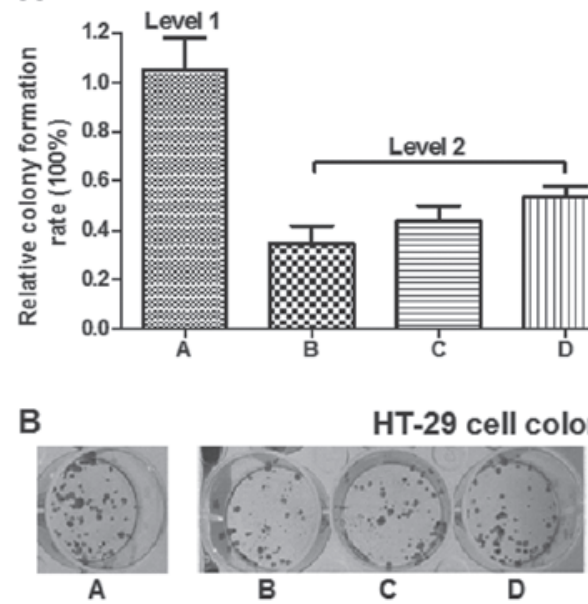

HT-29 cell colony in each group
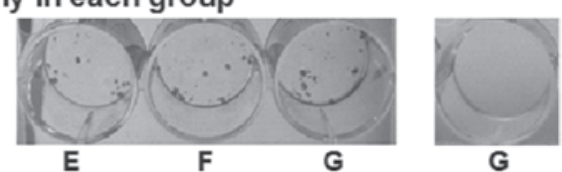

Figure 3. Effects of simultaneous knockdown of miR-183/96/182 on the colony formation rate of the HT-29 cells. (A) Similar to the MTT assay, eight groups of different concentrations and types of antisense oligonucleotide-miR mimics were transfected into the HT-29 cells. At $24 \mathrm{~h}$ after transfection, the HT-29 cells were seeded into a 12-well plate at a density of 200 cells/well and statistical analyses was performed to determine the colony formation rate in each group. (B) Representative images of the cells in each group following staining of the HT-29 cells. miR, microRNA. 

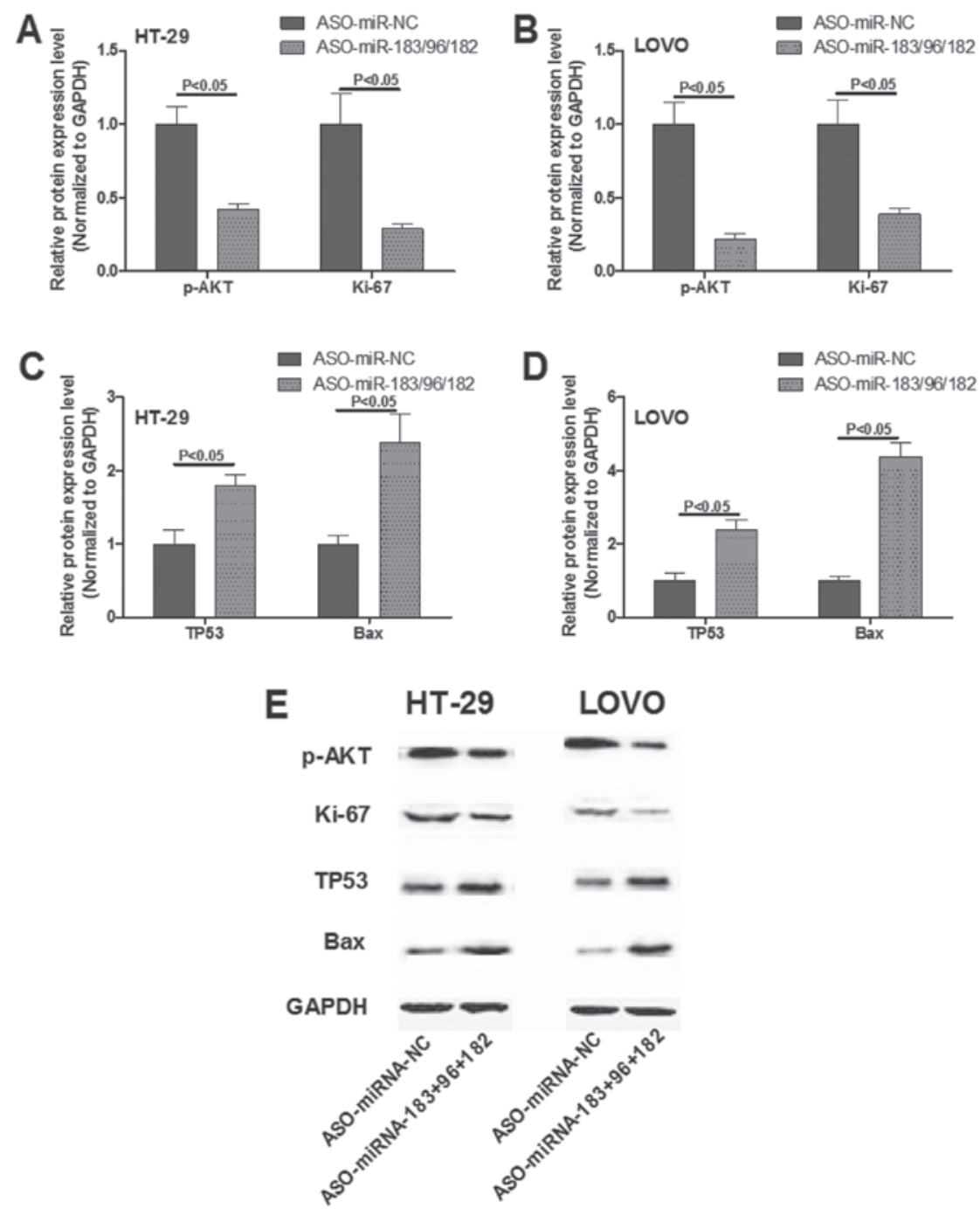

Figure 4. Effects of the miR-183/96/182 cluster on the expression of key proliferative- and apoptotic-associated proteins. (A-D) Western blot analysis was performed to detect the effect of miR-183/96/182 on the protein levels of p-AKT, Ki-67, Bax and TP53 in the HT-29 and LoVo cells, respectively. (E) Changes in the protein expression levels were measured using LabWork 4.0 software and normalized to GAPDH. $\mathrm{P}<0.05$ was considered to indicate a statistically significant difference. Each experiment was repeated three times and the representative images shown. miR, microRNA; ASO, antisense oligonucleotide; NC, negative control; p-AKT, phosphorylated-protein kinase B; TP53, tumor protein 53; Bax, B-cell-associated X protein.

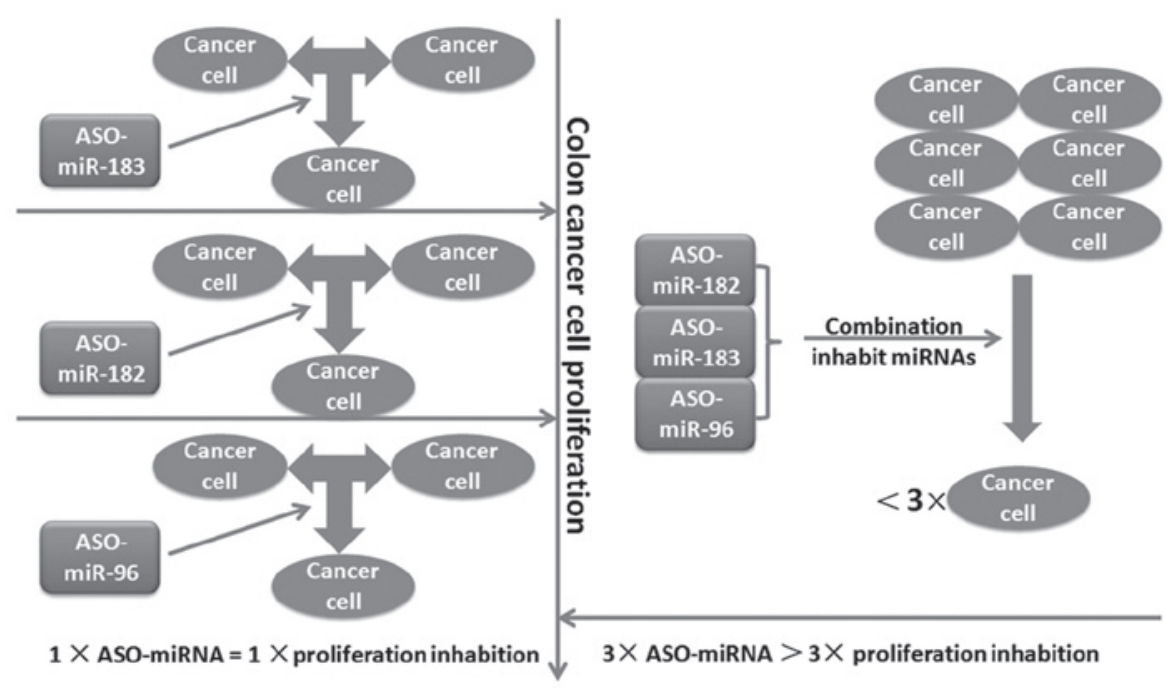

Figure 5. Schematic representation of the simultaneous knockdown of the expression of the miR-183/96/182 cluster efficiently inhibiting the proliferation of colon cancer cells compared with inhibiting them alone. On the left, each ASO miR in the miR-183/96/182 cluster was responsible for the inhibition of one colon cancer cell. On the right, three ASO miRs comprising all members of the miR-183/96/182 cluster caused over three times the inhibition of colon cancer cell proliferation. miR, microRNA; ASO, antisense oligonucleotide. 
Table II. Validated targets of miR-182/96/183 in the miRBASE database.

Validated targets

\begin{tabular}{|c|c|c|c|c|c|c|c|c|c|}
\hline \multicolumn{3}{|l|}{ miR-96 } & \multicolumn{3}{|c|}{ miR-182 } & \multicolumn{3}{|c|}{$\operatorname{miR}-183$} & \multirow{2}{*}{$\begin{array}{c}\text { miR-182/96 } \\
/ 183 \\
\text { ARRDC3 }\end{array}$} \\
\hline HIF1A & COX8A & VAMP8 & JAK2 & FOXO3 & TP53 & GFI1 & PRPH2 & TECTA & \\
\hline PAK3 & EIF2C1 & BNIP3L & IL17A & NFIB & EGR1 & HMOX1 & NFIB & BTRC & FOXO1 \\
\hline ARRDC3 & TBK1 & MYD88 & FOXO1 & PNLIP & EVI1 & FSCN1 & KLF4 & RET & ATOH1 \\
\hline ARIH2 & CISH & PSAT1 & HMGA2 & FBXW7 & ROS1 & RDX & GJB6 & IRS1 & DICER1 \\
\hline RNASEH2A & USF2 & MTSS1 & SAG & PRMT5 & COX8A & MSH6 & PIK3CA & EGR1 & NEUROD4 \\
\hline HTR1B & FOXO3 & HMG2L1 & BCL2L11 & RDX & ZEB2 & NEUROD4 & EIF2C1 & SOX2 & AKT1 \\
\hline RGS2 & FXR1 & POMC & MYC & RAC1 & ERBB2 & GEMIN4 & MYB & TWIST1 & PTEN \\
\hline FOXO1 & GFI1 & RHOD & BCL2 & MITF & PAK1 & MYO15A & AKAP12 & MLH1 & CASP2 \\
\hline STAT6 & LTBP1 & SLC12A5 & AKT1 & DOK4 & RNASEN & EGFR & KITLG & $\mathrm{C} 2$ & ZEB1 \\
\hline MYCN & GEMIN4 & BRAP & EGFR & ADCY6 & FRAP1 & IFI44 & DOK4 & HBEGF & TP53 \\
\hline ATOH1 & SLC1A1 & IL23A & ARRDC3 & CDKN1A & SLC1A1 & ATOH1 & BBS9 & ERBB2 & EIF2C2 \\
\hline HPRT1 & FBN1 & MYC & MYB & NAMPT & SLC7A5 & PI3 & TMC1 & RASA1 & COX8A \\
\hline DICER1 & MYBL1 & PITPNM1 & ENPP3 & $\mathrm{E} 2 \mathrm{~F} 3$ & CCND1 & TIA1 & BRAF & ZEB1 & EIF2C1 \\
\hline NEUROD4 & GEMIN5 & NCALD & DNTT & $\mathrm{CDC} 42$ & ZEB1 & MOS & PRB1 & MSH2 & CCND1 \\
\hline SSSCA1 & RIT2 & ADCY6 & MTSS1 & PAK3 & RGS17 & APC & CTNNB1 & CCL27 & MOS \\
\hline AKT1 & RNASEN & KRAS & KIT & SIRT1 & EIF2C3 & CASP2 & CCND1 & JAK2 & SAG \\
\hline HNF1A & IRS1 & TIA1 & SSSCA1 & BHLHB5 & ZNF828 & RNPC3 & ITGB1 & EZR & FBXW7 \\
\hline HOMER 1 & DKK2 & CREBZF & MYCN & BDNF & IRAK2 & SLC26A4 & DDX20 & EIF2C2 & DOK4 \\
\hline CAPNS1 & STAT3 & IL8 & MOCOS & NRIP1 & BAX & TNF & COL11A2 & ELSPBP1 & ADCY6 \\
\hline SOCS2 & BCR & SIRT1 & DICER1 & HBEGF & CCL27 & PDCD4 & TP53 & TIAM1 & MITF \\
\hline PTEN & SCPEP1 & FABP4 & EP300 & IL17F & IL2 & POU4F3 & FBXW7 & DFNB59 & \\
\hline TGFB1 & ZEB2 & PRMT5 & NEUROD4 & RARG & EIF2C2 & DICER1 & HMGA2 & MITF & \\
\hline KLK3 & FOSB & CCND1 & PRB1 & BRCA1 & CASP2 & SFRS2 & PTEN & STMN1 & \\
\hline CASP2 & EIF2C2 & MOS & PCNA & MOS & ATOH1 & SAG & COX8A & ATP8A2 & \\
\hline FRAP1 & ТВP & PTPRR & CREB1 & RELA & TNF & BIRC5 & NRIP1 & KRAS & \\
\hline ALK & SAG & DOK4 & CD38 & sox6 & CTTN & IDH2 & KIF2A & ARRDC3 & \\
\hline PRPH2 & BBC3 & SIP1 & PTEN & SLC12A2 & EIF2C1 & ADCY6 & GJB2 & AMACR & \\
\hline CDKN1A & MAPK8 & FSCN1 & & & & IGF2 bp1 & RHOD & NPC1 & \\
\hline FMR1 & MCL1 & FBXW7 & & & & NTRK1 & AKT1 & FOXO1 & \\
\hline BCL2L11 & CDKN1B & DDIT4 & & & & GRB2 & FBN1 & CA1 & \\
\hline ZEB1 & TP53 & SOX9 & & & & & & & \\
\hline GADD45A & EPB41L3 & $\mathrm{E} 2 \mathrm{~F} 3$ & & & & & & & \\
\hline DDX20 & CDH17 & CREB1 & & & & & & & \\
\hline PPIA & CACNA2D2 & MITF & & & & & & & \\
\hline MAP4K1 & NR3C1 & GPHN & & & & & & & \\
\hline
\end{tabular}

miR, microRNA.

(p)-Akt, Bax and TP53 were examined by western blot analysis. In the HT-29 and LoVo cells, the combined inhibition of the miR-183 cluster increased the activated expression of wild-type p53 and Bax and decreased the expression of p-Akt and the cell proliferation marker Ki-67 (Fig. 4). Collectively, the observation of induced apoptosis and decreased proliferation resulting from pooled knockdown of the miR-183 cluster in colon cells implied that treatment of colon cancer tumorigenesis using miRNA as a target in an miRNA-cluster-dependent manner may efficiently reduce colon cancer cell proliferation.

\section{Discussion}

miRNAs, $\sim 22 \mathrm{nt}$ in length, are a novel class of regulatory molecules with the ability to control the expression levels of thousands of genes and appear to decrease the expression of proteins by increasing the degradation or suppressing the translation of mRNA (22). Accumulating evidence indicates that miRNAs also function as oncogenes or tumor suppressor genes, which contribute to the tumorigenesis of several types of cancer, including colon cancer $(23,24)$. In the present study, five eligible studies containing 196 samples and 15 CRC cell lines were examined. As listed in Table I, several dysregulated miRNAs were found, and subsequent investigation focused on the miR-183/96/182 cluster. Based on the data in Table I, the average expression levels of miR-183, miR-96 and miR-182 increased 2.30-, 2.31- and 3.03-fold, respectively, in colon cancer tissues compared with normal tissue.

The human miR-183/96/182 cluster is located on human chromosome 7q32.2. The combined expression of these miRNAs may function in physiology and pathology, including tumor pathology. The human miR-183/96/182 cluster has been demonstrated as being overexpressed in several types of tumor and acting as an oncogene. Han et al (25) suggested 
that its overexpression is a marker for bladder cancer. Mihelich et al (26) identified the members of this cluster as having diagnostic and prognostic implications in prostate cancer. In addition, Yamada et al (27) identified two members of the cluster, miR-96 and miR-183 serve as potential tumor markers of urothelial carcinoma and Weeraratne et al (28) reported that the effects of the miR-183/96/182 cluster converge to regulate cell survival, proliferation and migration in medulloblastoma. The miR-183/96/182 cluster was also found to regulate oxidative apoptosis and sensitize cells to chemotherapy in gliomas (16,25-29). However, the effects of their coordinate expression on the mechanisms of tumorigenesis and particularly the proliferation of colon cancer remain to be fully elucidated. In the present study, this cluster was overexpressed in colon cancer, which was in accordance with a previous study (21). A series of transfection oligo-nucleotides were designed to detect the effects of the miR-183/96/182 cluster in colon cancer. Notably, these miRNAs were observed to coordinately regulate the proliferation of colon cancer cells with a synergistic effect, which was termed $1 \times$ ASO-miRNA $=1 \times$ cell proliferation inhibition in the present study, however $3 \times$ ASO-miRNA $>3$ x cell proliferation inhibition (Fig. 5).

The results of the present study indicated that the combined biological effects of the three miRNAs in the miR-182/96/183 cluster possessed increased inhibitory properties compared with each individual miRNA alone. They exhibited the same directional transcription and highly conserved 'seed sequences' and acted as a unit that significantly regulated the phonotype of the colon cancer cells, similar to the results observed by Tang et al in glioma (29). These findings provided support that miRNAs, which reside in clusters in the genome, function synergistically in cancer tumorigenesis. To further explain these mechanisms, the present study used the miRBASE database (http://www. mirbase.org/) to identify the targets of miR-182/96/183. As shown in Table II, 105 validated targets of miR-96, 81 targets of miR-182 and 90 targets of miR-183 were identified. However, only 20 targets were simultaneously targeted by all three miRNAs, which may explain why their simultaneous inhibition led to a synergistic increase in cell proliferation inhibition compared with inhibition of the miRNAs alone.

In conclusion, the present study demonstrated that increased expression of the miR-183/96/182 cluster was implicated in human colon cancer. Knockdown of the miR-183/96/182 cluster inhibited the survival of colon cancer cells and knockdown of the miR-183/96/182 cluster enhanced the anticancer proliferation effect more efficiently than knockdown of each alone. The co-expression of miRNA cluster ASOs may be a pleiotropic target for colon cancer therapy.

\section{References}

1. Weir HK, Thun MJ, Hankey BF, et al: Annual report to the nation on the status of cancer, 1975-2000, featuring the uses of surveillance data for cancer prevention and control. J Natl Cancer Inst 95: 1276-1299, 2003.

2. Greenlee RT, Murray T, Bolden S and Wingo PA: Cancer statistics, 2000. CA Cancer J Clin 50: 7-33, 2000.

3. Jemal A, Bray F, Center MM, et al: Global cancer statistics. CA Cancer J Clin 61: 69-90, 2011.

4. Fearon ER and Vogelstein B: A genetic model for colorectal tumorigenesis. Cell 61: 759-767, 1990.

5. Cho KR and Vogelstein B: Genetic alterations in the adenoma-carcinoma sequence. Cancer 70: 1727-1731, 1992.
6. Vogelstein B and Kinzler KW: The multistep nature of cancer. Trends Genet 9: 138-141, 1993.

7. Bhatti I,Lee A,Lund J and Larvin M: Small RNA: a large contributor to carcinogenesis? J Gastrointest Surg 13: 1379-1388, 2009.

8. Schetter AJ and Harris CC: Alterations of microRNAs contribute to colon carcinogenesis. Semin Oncol 38: 734-742, 2011.

9. Wu WK, Law PT, Lee CW, et al: MicroRNA in colorectal cancer: from benchtop to bedside. Carcinogenesis 32: 247-253, 2011.

10. Li H, Kloosterman W and Fekete DM: MicroRNA-183 family members regulate sensorineural fates in the inner ear. J Neurosci 30: 3254-3263, 2010.

11. Zhu Q, Sun W, Okano K, et al: Sponge transgenic mouse model reveals important roles for the microRNA-183 (miR-183)/96/182 cluster in postmitotic photoreceptors of the retina. J Biol Chem 286: 31749-31760, 2011.

12. Xu S, Witmer PD, Lumayag S, Kovacs B and Valle D: MicroRNA (miRNA) transcriptome of mouse retina and identification of a sensory organ-specific miRNA cluster. J Biol Chem 282: 25053-25066, 2007.

13. Krol J, Busskamp V, Markiewicz I, et al: Characterizing light-regulated retinal microRNAs reveals rapid turnover as a common property of neuronal microRNAs. Cell 141: 618-631, 2010.

14. Weston MD, Pierce ML, Jensen-Smith HC, et al: MicroRNA-183 family expression in hair cell development and requirement of microRNAs for hair cell maintenance and survival. Dev Dyn 240: 808-819, 2011.

15. Wu M, Huang C, Gan K, et al: LRRC4, a putative tumor suppressor gene, requires a functional leucine-rich repeat cassette domain to inhibit proliferation of glioma cells in vitro by modulating the extracellular signal-regulated kinase/protein kinase B/nuclear factor-kappaB pathway. Mol Biol Cell 17: 3534-3542, 2006.

16. Sarver AL, French AJ, Borralho PM, et al: Human colon cancer profiles show differential microRNA expression depending on mismatch repair status and are characteristic of undifferentiated proliferative states. BMC Cancer 9: 401, 2009.

17. Hamfjord J, Stangeland AM, Hughes T, et al: Differential expression of miRNAs in colorectal cancer: comparison of paired tumor tissue and adjacent normal mucosa using high-throughput sequencing. PLoS One 7: e34150, 2012.

18. Piepoli A1, Tavano F, Copetti M, et al: Mirna expression profiles identify drivers in colorectal and pancreatic cancers. PLoS One 7: e33663, 2012.

19. Arndt GM1, Dossey L, Cullen LM, et al: Characterization of global microRNA expression reveals oncogenic potential of miR-145 in metastatic colorectal cancer, BMC Cancer 9: 374, 2009.

20. Bandrés E1, Cubedo E, Agirre X, et al: Identification by Real-time PCR of 13 mature microRNAs differentially expressed in colorectal cancer and non-tumoral tissues. Mol Cancer 5: 29, 2006.

21. Sarver AL, Li L and Subramanian S: MicroRNA miR-183 functions as an oncogene by targeting the transcription factor EGR1 and promoting tumor cell migration. Cancer Res 70: 9570-9580, 2010.

22. Bartel DP: MicroRNAs: genomics, biogenesis, mechanism, and function. Cell 116: 281-297, 2004.

23. Gregersen LH, Jacobsen AB, Frankel LB, et al: MicroRNA-145 targets YES and STAT1 in colon cancer cells. PLoS One 5: e8836, 2010.

24. Tazawa $H$, Tsuchiya $N$, Izumiya $M$ and Nakagama $H$ : Tumor-suppressive miR-34a induces senescence-like growth arrest through modulation of the E2F pathway in human colon cancer cells. Proc Natl Acad Sci USA 104: 15472-15477, 2007.

25. Han Y, Chen J, Zhao X, et al: MicroRNA expression signatures of bladder cancer revealed by deep sequencing. PLoS One 6: e18286, 2011.

26. Mihelich BL, Khramtsova EA, Arva N, et al: miR-183-96-182 cluster is overexpressed in prostate tissue and regulates zinc homeostasis in prostate cells. J Biol Chem 286: 44503-44511, 2011.

27. Yamada Y, Enokida H, Kojima S, et al: MiR-96 and miR-183 detection in urine serve as potential tumor markers of urothelial carcinoma: correlation with stage and grade, and comparison with urinary cytology. Cancer Sci 102: 522-529, 2011.

28. Weeraratne SD, Amani V, Teider N, et al: Pleiotropic effects of miR-183 96 182 converge to regulate cell survival, proliferation and migration in medulloblastoma. Acta Neuropathol 123: 539-552, 2012.

29. Tang H,Bian Y, Tu C, et al: The miR-183/96/182 cluster regulates oxidative apoptosis and sensitizes cells to chemotherapy in gliomas. Curr Cancer Drug Targets 13: 221-231, 2013. 